\title{
Prevalence of sarcoidosis-associated pulmonary hypertension: cumulative analysis of two PULSAR studies
}

To the Editor:

We have read with great interest the study by Huitema et al. [1] showing a pulmonary hypertension $(\mathrm{PH})$ prevalence of around 3\% in a large cohort of predominantly Caucasian sarcoidosis patients in a Dutch tertiary centre. According to the authors, a European Caucasian cohort has never been studied before this PULmonary hypertension in pulmonary SARcoidosis (PULSAR) study. In this context, in 2013 we reported another PULSAR study (PULmonary hypertension in SARcoidosis) in an albeit smaller cohort from a German tertiary centre [2]. Of interest, we showed very similar results, with a prevalence of precapillary $\mathrm{PH}$ of $3.6 \%$ with a similar study design in Caucasian sarcoidosis patients. Taken together, we think that it is very remarkable that both PULSAR studies confirm consistent prevalences and a cumulative analysis of both PULSAR trials would demonstrate sarcoidosis-associated pulmonary hypertension (SAPH) proven by right heart catheter in 15/510 patients and precapillary PH in $13 / 510$ patients (table 1). The strength of both PULSAR studies is the prospective design and the invasive measurement of haemodynamics in most patients with suspicion of $\mathrm{PH}$ in transthoracic echocardiography; therefore, we would propose to use these algorithms to evaluate $\mathrm{PH}$ in sarcoidosis patients with unexplained dyspnoea. Echocardiography can show different signs of $\mathrm{PH}$, before the definitive diagnosis for PH should be made by right heart catheterisation. Regarding the advanced stages with fibrosis in a relevant part of SAPH patients in both studies, we think that in these Caucasian cohorts the obliteration of the vascular bed by parenchymal fibrosis is most often the cause; however, the role of different factors, including pulmonary vascular compression due to adenopathy, granulomatous arteritis, pulmonary veno-occlusive disease, portopulmonary hypertension and postcapillary $\mathrm{PH}$ due to cardiac sarcoidosis, remains open. As we know that $\mathrm{PH}$ is a complication of advanced pulmonary sarcoidosis and it is associated with increasing morbidity and mortality, it is important to increase the awareness of SAPH and also to identify these different phenotypes.

TABLE 1 Cumulative analysis of two PULSAR studies showing prevalence of sarcoidosis-associated $\mathrm{PH}$ among sarcoidosis patients

\begin{tabular}{lccc} 
Prevalence & PULSAR 2013 [2] & PULSAR 2019 [1] & Cumulative values \\
\hline PH suspected by echocardiography & $23 / 111(20.7 \%)$ & $28 / 399(7 \%)$ & $51 / 510(10 \%)$ \\
PH proven by right heart catheterisation & $5 / 111(4.5 \%)$ & $10 / 399(2.5 \%)$ & $15 / 510(2.9 \%)$ \\
Precapillary PH & $4 / 111(3.6 \%)$ & $9 / 399(2.3 \%)$ & $13 / 510(2.5 \%)$
\end{tabular}

PULSAR: PULmonary hypertension in (pulmonary) SARcoidosis; PH: pulmonary hypertension.

Treatment regimens were not assessed in both studies. Several drugs have been used to treat SAPH and have been investigated in different registries [3-6], showing that pulmonary arterial hypertension-specific treatment may be helpful in selected patients; however, controlled trials are rare. Next steps should be detailed phenotyping of SAPH patients and further studies that are needed to evaluate different treatment options in these selected SAPH phenotypes.

@ERSpublications

A cumulative analysis of both PULSAR trials would demonstrate sarcoidosis-associated pulmonary hypertension proven by right heart catheter in $15 / 510$ patients and precapillary $\mathrm{PH}$ in $13 / 510$ patients http://bit.ly/34VGWbv

Cite this article as: Pabst S, Grohé C, Skowasch D. Prevalence of sarcoidosis-associated pulmonary hypertension: cumulative analysis of two PULSAR studies. Eur Respir J 2020; 55: 1902223 [https://doi.org/ 10.1183/13993003.02223-2019]. 
Stefan Pabst ${ }^{1}$, Christian Grohé ${ }^{2}$ and Dirk Skowasch ${ }^{3}$

${ }^{1}$ Pneumologische Gemeinschaftspraxis Bonn, Bonn, Germany. ${ }^{2}$ Evangelische Lungenklinik Berlin, Berlin, Germany.

${ }^{3}$ Dept of Internal Medicine II - Pneumology/Cardiology, University Hospital Bonn, Bonn, Germany.

Correspondence: Dirk Skowasch, Dept of Internal Medicine II - Pneumology/Cardiology, University Hospital Bonn, Venusberg-Campus 1, 53127 Bonn, Germany. E-mail: dirk.skowasch@ukb.uni-bonn.de

Received: 18 Nov 2019 | Accepted: 17 Dec 2019

Conflict of interest: None declared.

\section{References}

1 Huitema MP, Bakker ALM, Mager JJ, et al. Prevalence of pulmonary hypertension in pulmonary sarcoidosis: the first large European prospective study. Eur Respir J 2019; 54: 1900897.

2 Pabst S, Hammerstingl C, Grau N, et al. Pulmonary arterial hypertension in patients with sarcoidosis: the Pulsar single center experience. Adv Exp Med Biol 2013; 755: 299-305.

3 Skowasch D, Huscher D, Pizarro C, et al. Sarcoidosis-associated pulmonary hypertension versus idiopathic pulmonary arterial hypertension: results from the COMPERA registry. Clin Res Cardiol 2016; 105: Suppl. 1, P1798.

4 Boucly A, Cottin V, Nunes $\mathrm{H}$, et al. Management and long-term outcomes of sarcoidosis-associated pulmonary hypertension. Eur Respir J 2017; 50: 1700465.

5 Baughman RP, Shlobin OA, Wells AU, et al. Clinical features of sarcoidosis associated pulmonary hypetension: results of a multi-national registry. Repir Med 2018; 139: 72-78.

6 Parikh KS, Dahhan T, Nicholl L, et al. Clinical features and outcomes of patients with sarcoidosis associated pulmonary hypertension. Sci Rep 2019: 9: 4061. 